\title{
Der Einfluss des Inhalationsrauchens auf die Klinik J. Schildge und die bronchoalveoläre Lavage bei Sarkoidose
}

\author{
The Influence of Smoking on Clinical Manifestation and Composition \\ of Bronchoalveolar Lavage in Sarcoidosis
}

\section{Zusammenfassung}

Die Sarkoidose ist definiert als entzündliche Systemerkrankung, deren histologisches Bild von Epitheloidzellgranulomen dominiert wird. In der bronchoalveolären Lavage (BAL) herrscht eine lymphozytäre Alveolitis mit erhöhtem CD4/CD8-Quotienten vor. Eine höhere Frequenz der Sarkoidose bei Nichtrauchern (NR) als bei Rauchern $(\mathrm{R})$ wird mitgeteilt. Die vorliegende Arbeit untersucht eventuelle Einflüsse des Inhalationsrauchens auf demographische Daten, Spirometrie und Resultate der BAL von 155 prospektiv diagnostizierten Patienten mit Sarkoidose. Verglichen wurden 111 NR (Stadium I/II+III 48/63) mit 44 R (23/21). Als Kontrolle dienten 16 Patienten ohne nachweisbare bronchopulmonale Erkrankung (9 NR, 7 R). Patienten mit Sarkoidose waren weniger häufig $R$ als NR ( 28 vs. $72 \%, p=0,0001$; Kontrollen 44 vs. $\left.56 \%, p=0,6\left[\chi^{2}\right]\right)$. Sarkoidose-R waren jünger als Sarkoidose-NR ( $40,4 \pm 11,9$ vs. $45,6 \pm 14,7$ Jahre, $p=0,009)$. Unterschiede in der IVC (\%Soll) bestanden nicht. Dennoch war bei Sarkoidose unter den R ein nachteiliger Effekt des Rauchens auf den Verlauf der IVC (\%Soll) mit zunehmendem Alter festzustellen, der bei NR nicht bestand ( $R$ vs. NR $r=-0,54, p=0,001$ vs. $r=-0,13, p=0,22$ ). In der BAL zeigten Sarkoidose-R einen geringeren Gehalt an Albumin (mg/dl) als Sarkoidose-NR (R vs. NR 9,5 $\pm 5,9$ vs. 14,5 \pm 13,4, $\mathrm{p}=0,012$; und einen Trend zu einer geringer ausgeprägten Lymphozytose (in \% der BAL-Zellen), R vs. NR 29,2 $\pm 21,1$ vs. $34,1 \pm 18,6, p=0,099$. Einflüsse des Rauchens auf die Lymphozytenpopulationen bei Sarkoidose waren nicht nachweisbar (CD4/CD 8-Quotient R vs. NR 10,0 $\pm 11,4$ vs. 7,2 $\pm 7,1, p=0,25$ ).

\section{Abstract}

Sarcoidosis is defined as an inflammatory systemic disease; the characteristic morphological feature is the noncaseating granuloma. Typical finding in bronchoalveolar lavage (BAL) is a lymphocytic alveolitis with an increased CD4/CD8-quotient. A higher frequency of sarcoidosis in non-smokers (NS) than in smokers $(S)$ has been reported. The influence of inhalative smoking on demographical data, lung function and results of BAL in prospectively selected patients with sarcoidosis was investigated. 111 NS (Sarcoidosis stage I/II+III 48/63) and 44 S (23/21) were included in the study. 16 patients without provable pulmonary disease (9 NS, 7 S) served as controls. Patients with sarcoidosis ware less often $S$ than NS ( 28 vs. $72 \%, p=0,0001$ ) Controls 44 vs. $\left.56 \%, p=0,6\left[\chi^{2}\right]\right)$. Sarcoidosis S were younger than NS $(40,4 \pm 11,9$ vs. $45,6 \pm 14,7$ years, $p=0,009)$. There were no differences in the IVC (in \% predicted). There was a negative effect of smoking on the course of the IVC (\% predicted) with incremental age, not seen in the non smoking group ( $\mathrm{S}$ vs. NS: $\mathrm{r}=-0,54, \mathrm{p}=0,001$ vs. $r=-0,13, p=0,22)$. In BAL of Sarcoidosis $S$ there was a lower concentration of albumin than of NS (in $\mathrm{mg} / \mathrm{dl}$ ), S vs. NS: 9,5 $\pm 5,9$ vs. $14,5 \pm 13,4, p=0,012$ and a trend to a less intensive lymphocytic alveolitis (in \% of BAL-cells, S vs. NS: $29,2 \pm 21,1$ vs. $34,1 \pm 18,6$, $\mathrm{p}=0,099$ ). Influences of the smoking on the populations of T-lymphocytes could not be seen. (CD4/CD8-ratio S vs. NS $10,0 \pm 11,4$ vs. $7,2 \pm 7,1, p=0,25)$. In conclusion patients with sarcoidosis were more often NS than S. S were younger than NS. A protective effect of smoking on the course of lung function in sarLehrkrankenhaus der Albert-Ludwigs-Universität Freiburg i.Br. der Pneumologie an den St. Vincentius-Kliniken Karlsruhe

Korrespondenzadresse Dr. med. Johannes Schildge · St. Vincentius-Kliniken Karlsruhe gAG Medizinische Klinik - Abteilung Pneumologie · Südendstraße 32 · 76137 Karlsruhe E-mail: johannes.schildge@vincentius-ka.de ·Internet: www.vincentius-ka.de 
Schlussfolgerung: Patienten mit Sarkoidose waren häufiger NR als R. Bei R war eine frühere Manifestation oder Erkennung der Sarkoidose belegbar. Hinweise auf einen protektiven Effekt des Rauchens auf die Ventilation ergaben sich nicht. R zeigten in der BAL mit einem geringeren Albumingehalt und einem Trend zu einer geringer ausgeprägten Lymphozytose Hinweise auf eine geringer ausgeprägte Alveolitis als NR. Einflüsse des Rauchens auf die Verteilung der Lymphozytenpopulationen waren nicht zu sehen. coidosis could be excluded. In the BAL S demonstrated a lower content of albumin and a trend to a less pronounced lymphocytosis and therefore a less pronounced alveolitis than NS. Influences of smoking on the distribution of the lymphocytic populations were not seen.

\section{Einleitung}

Die Sarkoidose ist charakterisiert als eine entzündliche Systemerkrankung unklarer Ursache, deren histologisches Entzündungsbild von nicht nekrotisierenden Epitheloidzellgranulomen bestimmt wird und die meist von einer pulmonalen Manifestation begleitet ist [1]. Inhalationsrauchen führt zu einer Entzündung der Atemwege und kann Immunvorgänge der Lunge beeinflussen. So gibt es einerseits entzündliche interstitielle Lungenerkrankungen, die regelhaft mit dem Inhalationsrauchen korreliert sind wie die respiratorische Bronchiolitis mit interstitieller Lungenerkrankung (RBILD), die desquamative interstitielle Pneumonie (DIP) oder die Histiocytosis X [2]. Bei Goodpasture's Syndrom disponiert Rauchen zu alveolärer Hämorrhagie [3]. Andererseits werden Sarkoidose und exogen allergische Alveolitis gehäuft bei Nichtrauchern beschrieben [4]. Als mögliche immunmodulierende Effekte des Rauchens werden eine Hemmung der Interleukin-Bildung der Alveolarmakrophagen [5], Suppression der CD4-Lymphozyten und Stimulation der CD8-Lymphozyten diskutiert [6-8].

Die bronchoalveoläre Lavage (BAL) ist ein bei der Diagnostik interstitieller Lungenerkrankungen etabliertes Verfahren. Oft ergeben sich diagnostisch relevante, gelegentlich aber auch beweisende Befunde. Insbesondere zum Nachweis einer Sarkoidose wird eine lymphozytäre Alveolitis mit Prädominanz von CD4-Zellen in der BAL bei typischem Röntgenbild als diagnosesichernd angesehen, auch wenn histologisch eine epitheloidzelliggranulomatöse Entzündung nicht belegt ist [9].

Wir untersuchten, ob und auf welche Weise das Inhalationsrauchen die klinische Manifestation und die Resultate der BAL von Patienten mit Sarkoidose beeinflusst.

\section{Methoden}

\section{Patienten}

In die Auswertung aufgenommen wurden 155 Patienten mit Sarkoidose, bei denen zwischen dem 1. Januar 1999 und dem 31.10. 2002 in der Abteilung Pneumologie der St. Vincentius-Kliniken unter ambulanten Bedingungen mittels Bronchoskopie, BAL und transbronchialer Lungenbiopsie (TBB) eine Sarkoidose nachgewiesen wurde. Die epidemiologischen Daten, qualitative sowie quantitative Nikotinanamnese und Röntgenstadien gehen aus Tab. 1 hervor. In der Auswertung wurden lebenslange Nichtraucher verglichen mit Patienten, die zum Zeitpunkt der BAL aktiven Nikotinkonsum in Form von Zigarettenrauchen in einer
Menge von über 10 Zigaretten pro Tag betrieben und seit mindestens 3 Jahren Raucher waren. Exraucher und Raucher unter 10 Zigaretten pro Tag wurden von der Untersuchung ausgeschlossen. Keiner der Patienten stand zum Zeitpunkt der Untersuchung unter einer immunsuppressiven Therapie. In die Studie aufgenommen wurden nur Patienten, deren Rückgewinn bei der BAL $>30 \%$ lag und bei denen der histologische Nachweis epitheloidzelliger Granulome im Lungengewebe oder in der Bronchialschleimhaut gelang.

Vor der Bronchoskopie wurde eine Fluss-Volumen-Kurve ${ }^{1}$ gemessen. Die Stadieneinteilung erfolgte nach radiologischen Kriterien aus der Thorax-Übersichtsaufnahme: Stadium I = isolierte Hiluslymphome ( $\mathrm{n}=71$ ), Stadium II = Hiluslymphome mit Parenchymbefall $(n=38)$, Stad. III = Parenchymbefall ohne Hiluslymphome $(n=46)$.

Als Kontrolle dienten 16 Patienten, die aufgrund einer über mehrere Monate anhaltenden nicht zu klärenden Reizhustensymptomatik untersucht wurden, bei denen sich aber klinisch, spirometrisch, in Thoraxübersichtsaufnahme und CT der Lunge in Dünnschichttechnik, in BAL und TBB kein pathologischer Befund ergab.

\section{Bronchoskopie, BAL, TBB}

Die Bronchoskopie wurde mit einem flexiblen Bronchoskop in Video- oder Fiberglastechnik ${ }^{2}$ unter pulsoxymetrischer Überwachung und Sauerstoffapplikation über Nasensonde durchgeführt. Nach intravenöser Prämedikation mit 5-7,5 mg Midazolam wurde das Bronchoskop unter lokaler Anästhesie mit maximal $20 \mathrm{ml}$ 2\%iger Xylocainlösung inhalativ und über den Instrumentierkanal transoral eingeführt. Zur BAL wurde die Bronchoskopspitze in einem Subsegmentbronchus des Mittellappens in wedge-Position gebracht und über einen im Instrumentierkanal liegenden Kunststoffkatheter $100 \mathrm{ml}$ isotonische, sterile, im Wasserbad auf $37^{\circ} \mathrm{C}$ erwärmte Kochsalzlösung in Portionen zu $20 \mathrm{ml}$ über eine Einmalspritze aus Kunststoff instilliert und sofort in die Spritze reaspiriert. Die Menge des Rückgewinns wurde festgehalten. Es wurden bei jedem Patient mindestens zwei TBB entnommen, daneben eine Schleimhautbiopsie aus der Oberlappencarina rechts.

\footnotetext{
${ }^{1}$ MasterLAB Fa. Jaeger \& Toennies, Höchberg

2 Olympus Optical Co. GmbH Hamburg
} 
Tab. 1 Patienten: Stadien, Demografische Daten, Nikotinanamnese, Lungenfunktion. BAL: humorale und zelluläre Eigenschaften. Mittelwerte mit Standardabweichung. $\mathrm{R}=$ Raucher, NR = Nichtraucher. $\mathrm{P}=$ Vergleich R mit NR (Mann-Whitney-U-Test;. ${ }^{*}=\chi^{2}$-Test)

\begin{tabular}{|c|c|c|c|c|c|c|c|c|c|c|c|c|c|c|}
\hline & \multicolumn{4}{|c|}{ Kontrolle } & \multicolumn{3}{|c|}{ Sarkoidose I } & \multicolumn{3}{|c|}{ Sarkoidose II und III } & \multicolumn{4}{|c|}{ Sarkoidose alle Stadien } \\
\hline & alle & $N R$ & $R$ & $p$ & alle & $N R$ & $R$ & alle & $N R$ & $R$ & alle & $N R$ & $R$ & $P$ \\
\hline$N($ davon + ) & $\begin{array}{l}16 \\
(7)\end{array}$ & $\begin{array}{l}9 \\
(5)\end{array}$ & $\begin{array}{l}7 \\
(2)\end{array}$ & $\begin{array}{l}0,6^{*} \\
(0,41)\end{array}$ & $\begin{array}{l}71 \\
(28)\end{array}$ & $\begin{array}{l}48 \\
(21)\end{array}$ & $\begin{array}{l}23 \\
(7)\end{array}$ & $\begin{array}{l}84 \\
(40)\end{array}$ & $\begin{array}{l}63 \\
(33)\end{array}$ & $\begin{array}{l}21 \\
(7)\end{array}$ & $\begin{array}{l}155 \\
(68)\end{array}$ & $\begin{array}{l}111 \\
(54)\end{array}$ & $\begin{array}{l}44 \\
(14)\end{array}$ & $\begin{array}{l}0,000^{*} \\
(0,058)\end{array}$ \\
\hline Alter (Jahre) & $\begin{array}{l}49,7 \\
\pm \\
12,7\end{array}$ & $\begin{array}{l}50,8 \\
\pm \\
11,9\end{array}$ & $\begin{array}{l}48,3 \\
\pm \\
14,4\end{array}$ & 0,92 & $\begin{array}{l}40,9 \\
\pm \\
13,6\end{array}$ & $\begin{array}{l}43,4 \\
\pm \\
14,1\end{array}$ & $\begin{array}{l}35,7 \\
\pm \\
10,9\end{array}$ & $\begin{array}{l}46,8 \\
\pm \\
14,1\end{array}$ & $\begin{array}{l}47,3 \\
\pm \\
15,1\end{array}$ & $\begin{array}{l}45,5 \\
6 \pm \\
11,1\end{array}$ & $\begin{array}{l}44,1 \\
\pm \\
14,1\end{array}$ & $\begin{array}{l}45,6 \\
\pm \\
14,7\end{array}$ & $\begin{array}{l}40,4 \\
\pm \\
11,9\end{array}$ & 0,009 \\
\hline Packyears & & & $\begin{array}{l}32,1 \\
\pm \\
18,5\end{array}$ & & & & $\begin{array}{l}13,1 \\
\pm \\
11,6\end{array}$ & & & $\begin{array}{l}28,2 \\
\pm \\
19,1\end{array}$ & & & $\begin{array}{l}20,2 \\
\pm \\
17,3\end{array}$ & \\
\hline IVC \%soll & $\begin{array}{l}101,0 \\
\pm \\
11,3\end{array}$ & $\begin{array}{l}102,7 \\
\pm \\
13,4\end{array}$ & $\begin{array}{l}98,9 \\
\pm \\
8,2\end{array}$ & 0,54 & $\begin{array}{l}95,5 \\
\pm \\
13,1\end{array}$ & $\begin{array}{l}95,8 \\
\pm \\
14,4\end{array}$ & $\begin{array}{l}95,0 \\
\pm \\
10,0\end{array}$ & $\begin{array}{l}79,1 \\
\pm \\
16,4\end{array}$ & $\begin{array}{l}79,2 \\
\pm \\
14,9\end{array}$ & $\begin{array}{l}78,6 \\
\pm \\
21,3\end{array}$ & $\begin{array}{l}86,8 \\
\pm \\
17,0\end{array}$ & $\begin{array}{l}86,6 \\
\pm \\
16,8\end{array}$ & $\begin{array}{l}87,6 \\
\pm \\
17,8\end{array}$ & 0,45 \\
\hline FEV ${ }^{1} \%$ soll & $\begin{array}{l}101,7 \\
\pm \\
11,3\end{array}$ & $\begin{array}{l}105,9 \\
\pm \\
11,6\end{array}$ & $\begin{array}{l}96,3 \\
\pm \\
9,6\end{array}$ & 0,09 & $\begin{array}{l}96,3 \\
\pm \\
15,2\end{array}$ & $\begin{array}{l}95,7 \\
\pm \\
16,8\end{array}$ & $\begin{array}{l}97,7 \\
\pm \\
11,5\end{array}$ & $\begin{array}{l}78,0 \\
\pm \\
17,6\end{array}$ & $\begin{array}{l}78,8 \\
\pm \\
17,2\end{array}$ & $\begin{array}{l}75,5 \\
\pm \\
19,2\end{array}$ & $\begin{array}{l}86,7 \\
\pm \\
18,8\end{array}$ & $\begin{array}{l}86,2 \\
\pm \\
18,9\end{array}$ & $\begin{array}{l}87,8 \\
\pm \\
18,8\end{array}$ & 0,51 \\
\hline Rückgewinn (\%) & $\begin{array}{l}59,0 \\
\pm \\
11,8\end{array}$ & $\begin{array}{l}61,3 \\
\pm \\
8,9\end{array}$ & $\begin{array}{l}56,0 \\
\pm \\
15,1\end{array}$ & 0,76 & $\begin{array}{l}62,9 \\
\pm \\
9,5\end{array}$ & $\begin{array}{l}63,1 \\
\pm \\
11,1\end{array}$ & $\begin{array}{l}62,8 \\
\pm \\
8,7\end{array}$ & $\begin{array}{l}61,5 \\
\pm \\
11,5\end{array}$ & $\begin{array}{l}62,6 \\
\pm \\
10,9\end{array}$ & $\begin{array}{l}58,1 \\
\pm \\
12,8\end{array}$ & $\begin{array}{l}62,1 \\
\pm \\
10,6\end{array}$ & $\begin{array}{l}62,7 \\
\pm \\
10,0\end{array}$ & $\begin{array}{l}60,7 \\
\pm \\
12,0\end{array}$ & 0,58 \\
\hline Albumin (mg/dl) & $\begin{array}{l}4,1 \\
\pm \\
2,3\end{array}$ & $\begin{array}{l}4,9 \\
\pm \\
2,6\end{array}$ & $\begin{array}{l}3,2 \\
\pm \\
1,6\end{array}$ & 0,25 & $\begin{array}{l}11,6 \\
\pm \\
9,8\end{array}$ & $\begin{array}{l}13,5 \\
\pm \\
10,9\end{array}$ & $\begin{array}{l}7,5 \\
\pm \\
4,9\end{array}$ & $\begin{array}{l}14,4 \\
\pm \\
13,4\end{array}$ & $\begin{array}{l}15,2 \\
\pm \\
15,0\end{array}$ & $\begin{array}{l}11,7 \\
\pm \\
6,3\end{array}$ & $\begin{array}{l}13,1 \\
\pm \\
12,0\end{array}$ & $\begin{array}{l}14,5 \\
\pm \\
13,4\end{array}$ & $\begin{array}{l}9,5 \\
\pm \\
5,9\end{array}$ & 0,012 \\
\hline Zellen $\left({ }^{*} 10^{6}\right)$ & $\begin{array}{l}9,5 \\
\pm \\
5,0\end{array}$ & $\begin{array}{l}9,7 \\
\pm \\
5,3\end{array}$ & $\begin{array}{l}9,1 \\
\pm \\
5,0\end{array}$ & 0,96 & $\begin{array}{l}15,8 \\
\pm \\
9,1\end{array}$ & $\begin{array}{l}16,5 \\
\pm \\
9,5\end{array}$ & $\begin{array}{l}14,5 \\
\pm \\
8,2\end{array}$ & $\begin{array}{l}17,0 \\
\pm \\
11,0\end{array}$ & $\begin{array}{l}16,1 \\
\pm \\
11,1\end{array}$ & $\begin{array}{l}20,0 \\
\pm \\
10,0\end{array}$ & $\begin{array}{l}16,5 \\
\pm \\
10,1\end{array}$ & $\begin{array}{l}16,2 \\
\pm \\
10,4\end{array}$ & $\begin{array}{l}17,1 \\
\pm \\
9,4\end{array}$ & 0,42 \\
\hline Makrophagen (\%) & $\begin{array}{l}94,4 \\
\pm \\
4,0\end{array}$ & $\begin{array}{l}94,2 \\
\pm \\
4,4\end{array}$ & $\begin{array}{l}94,6 \\
\pm \\
3,7\end{array}$ & 0,92 & $\begin{array}{l}65,4 \\
\pm \\
18,7\end{array}$ & $\begin{array}{l}62,3 \\
\pm \\
19,1\end{array}$ & $\begin{array}{l}74,6 \\
\pm \\
18,8\end{array}$ & $\begin{array}{l}65,4 \\
\pm \\
18,7\end{array}$ & $\begin{array}{l}63,5 \\
\pm \\
19,4\end{array}$ & $\begin{array}{l}65,6 \\
\pm \\
19,7\end{array}$ & $\begin{array}{l}64,1 \\
\pm \\
18,9\end{array}$ & $\begin{array}{l}64,1 \\
\pm \\
18,9\end{array}$ & $\begin{array}{l}69,3 \\
\pm \\
21,5\end{array}$ & 0,085 \\
\hline Lymphozyten (\%) & $\begin{array}{l}4,9 \\
\pm \\
3,3\end{array}$ & $\begin{array}{l}4,9 \\
\pm \\
3,5\end{array}$ & $\begin{array}{l}4,9 \\
\pm \\
3,4\end{array}$ & 0,92 & $\begin{array}{l}32,5 \\
\pm \\
20,6\end{array}$ & $\begin{array}{l}36,4 \\
\pm \\
18,9\end{array}$ & $\begin{array}{l}24,5 \\
\pm \\
22,1\end{array}$ & $\begin{array}{l}32,9 \\
\pm \\
18,4\end{array}$ & $\begin{array}{l}32,4 \\
\pm \\
18,3\end{array}$ & $\begin{array}{l}34,3 \\
\pm \\
19,1\end{array}$ & $\begin{array}{l}32,7 \\
\pm \\
19,4\end{array}$ & $\begin{array}{l}34,1 \\
\pm \\
18,6\end{array}$ & $\begin{array}{l}29,2 \\
\pm \\
21,1\end{array}$ & 0,099 \\
\hline Neutrophile (\%) & $\begin{array}{l}0,5 \\
\pm \\
0,7\end{array}$ & $\begin{array}{l}0,6 \\
\pm \\
0,8\end{array}$ & $\begin{array}{l}0,4 \\
\pm \\
0,4\end{array}$ & 0,76 & $\begin{array}{l}0,73 \\
\pm \\
0,85\end{array}$ & $\begin{array}{l}0,74 \\
\pm \\
0,91\end{array}$ & $\begin{array}{l}0,73 \\
\pm \\
0,75\end{array}$ & $\begin{array}{l}1,10 \\
\pm \\
1,87\end{array}$ & $\begin{array}{l}1,14 \\
\pm \\
2,10\end{array}$ & $\begin{array}{l}0,96 \\
\pm \\
0,90\end{array}$ & $\begin{array}{l}0,93 \\
\pm \\
1,50\end{array}$ & $\begin{array}{l}0,97 \\
\pm \\
1,70\end{array}$ & $\begin{array}{l}0,84 \\
\pm \\
0,82\end{array}$ & 0,52 \\
\hline Eosinophile (\%) & $\begin{array}{l}0,2 \\
\pm \\
0,3\end{array}$ & $\begin{array}{l}0,3 \\
\pm \\
0,4\end{array}$ & $\begin{array}{l}0,1 \\
\pm \\
0,2\end{array}$ & 0,81 & $\begin{array}{l}0,38 \\
\pm \\
0,65\end{array}$ & $\begin{array}{l}0,46 \\
\pm \\
0,72\end{array}$ & $\begin{array}{l}0,22 \\
\pm \\
0,42\end{array}$ & $\begin{array}{l}0,75 \\
\pm \\
1,05\end{array}$ & $\begin{array}{l}0,69 \\
\pm \\
1,04\end{array}$ & $\begin{array}{l}0,95 \\
\pm \\
1,05\end{array}$ & $\begin{array}{l}0,58 \\
\pm \\
0,90\end{array}$ & $\begin{array}{l}0,59 \\
\pm \\
0,92\end{array}$ & $\begin{array}{l}0,57 \\
\pm \\
0,86\end{array}$ & 0,55 \\
\hline
\end{tabular}

\section{BAL-Analytik}

Nach Entnahme von $5 \mathrm{ml}$ zur mikrobiologischen Analytik wurde die BAL durch Filtration über Gaze ${ }^{3}$ von groben Partikeln befreit und in einem sterilen Kunststoffgefäß gepoolt. Die Gesamtzellzahl wurde an einem Zellanalysegerät ${ }^{4}$ durchflusszytometrisch bestimmt. Zellpräparate wurden durch Zentrifugation ${ }^{5}$ angefertigt. Die prozentuale Verteilung der Zellgruppen wurde durch mikroskopische Auszählung von 500 Zellen eines nach MayGrünwald-Giemsa gefärbten Präparates ermittelt. Bei einem Lymphozytenanteil von über $15 \%$ wurde die prozentuale Verteilung der Lymphozytenoberflächeneigenschaften CD3 (T-Zellen), CD4 (T-Helfer-Zellen), CD8 (T-Suppressor-Zellen) bestimmt und der CD4/CD8-Quotient errechnet. Als Sekundärantikörper diente der Alkalische Phosphatase-Anti-Alkalische Phosphatase-Antikörper ${ }^{6}$.

\footnotetext{
${ }^{3}$ Topper 8, Fa. Johnson \& Johnson, Norderstedt

${ }^{4} \mathrm{~K} 800 \mathrm{Fa}$. Sysmex, Norderstedt

${ }^{5}$ Cytospin 2, Fa. Shandon, Frankfurt a.M.

${ }^{6}$ Dako, Hamburg
}

Der Albumingehalt in der BAL als Hinweis auf eine entzündliche Exsudation wurde über ein käufliches Testverfahren immunologisch durch Einsatz von Kaninchenantiserum und anschließender vollautomatischer Messung am Nephelometer BN $100^{7}$ bestimmt.

\section{Statistik}

Die Prüfung der Messreihen auf Normalverteilung mithilfe des Kolmogorov-Smirnov-Tests zeigte, dass die meisten Variablen nicht normalverteilt sind. Aus diesem Grunde kamen nichtparametrische Tests zur Anwendung. Unterschiede zwischen Rauchern und Nichtrauchern mit Sarkoidose wurden mit dem Mann-Whithney-U-Test und dem $\chi^{2}$-Test untersucht. Korrelationen verschiedener Variablen wurden mit der nichtparametrischen Korrelation nach Spearman errechnet. Mehrfachvergleiche erfolgten nach Tukey, wobei Patienten ohne und mit Lungenparenchymbefall getrennt betrachtet und somit Stadium II und III zu einer Gruppe zusammengefasst wurden. Eine weitere Unterteilung war wegen zu geringer Belegung der Untergruppen

${ }^{7}$ Dade Behring, Marburg 


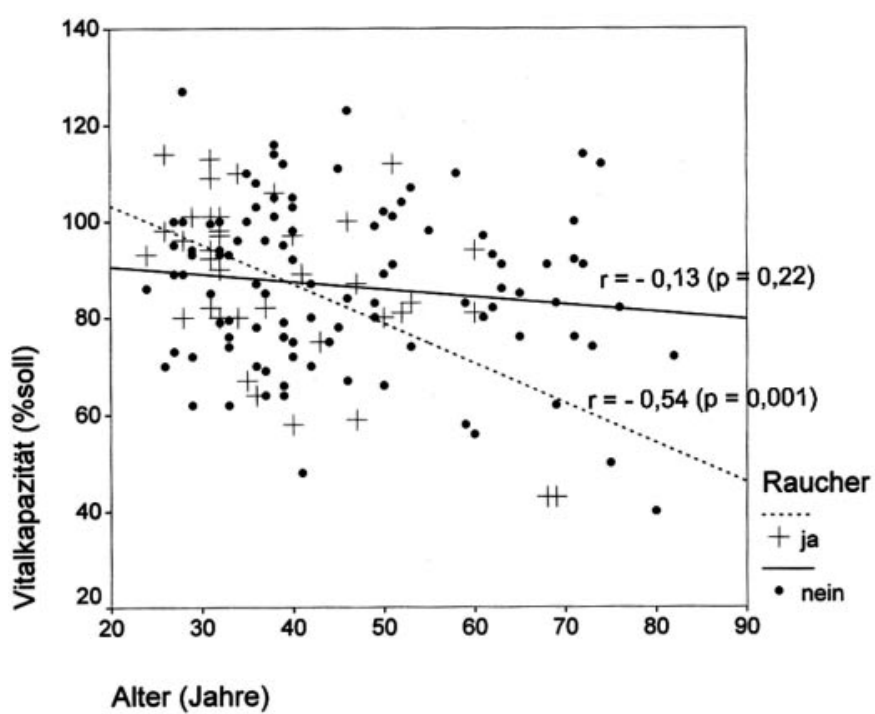

Abb. 1 Korrelation des Lebensalters mit der IVC in \% Soll. $r=$ Korrelationskoeffizient nach Spearman. Ohne Kontrollen. Die bei R nachweisbare negative Korrelation ist bei NR nicht vorhanden.

und stark unterschiedlicher Gruppengrößen statistisch nicht sinnvoll. Eine Irrtumswahrscheinlichkeit unter $5 \%$ wurde als signifikant definiert.

\section{Ergebnisse}

\section{Demografische und klinische Parameter (Tab. 1)}

Der Anteil der Raucher betrug unter den Patienten mit Sarkoidose $28 \%$ und war im Gegensatz zur Kontrollgruppe signifikant kleiner als der Nichtraucheranteil. 21\% der weiblichen und 34\% der männlichen Patienten waren Raucher. Das Überwiegen der Männer unter den Rauchern war zwar nicht signifikant, zeigte aber einen Trend $(p=0,058)$.

Nichtraucher waren im Mittel 5 Jahre älter als die Raucher, der Altersunterschied war signifikant.

Bei den spirometrischen Leitgrößen, der IVC und der $\mathrm{FEV}_{1}$, war kein Unterschied zwischen Rauchern und Nichtrauchern festzustellen. Bei der Korrelation der IVC (in \% Soll) mit dem Lebensalter zeigte sich bei den Nichtrauchern keine signifikante Beziehung. Im Gegensatz hierzu nahm bei den Rauchern die IVC mit zunehmendem Lebensalter signifikant ab (Abb.1).

\section{BAL-Befunde (Tab. 2)}

Der Rückgewinn, die Gesamtzellzahl und die Differenzialzytologie waren zwischen Rauchern und Nichtrauchern nicht verschieden. Es zeigte sich allerdings ein Trend zu einem höheren relativen Makrophagenanteil $(p=0,085)$ und einem geringeren relativen Lymphozytenanteil $(p=0,099)$ bei Rauchern. Im Untergruppenvergleich zeigten Patienten mit Sarkoidose II + III, unabhängig vom Rauchverhalten, einen im Vergleich mit den Kontrollen signifikant erhöhten Lymphozytenanteil. Im Stadium I war diese Beziehung nur bei Nichtrauchern statistisch belegbar (Abb. 2).

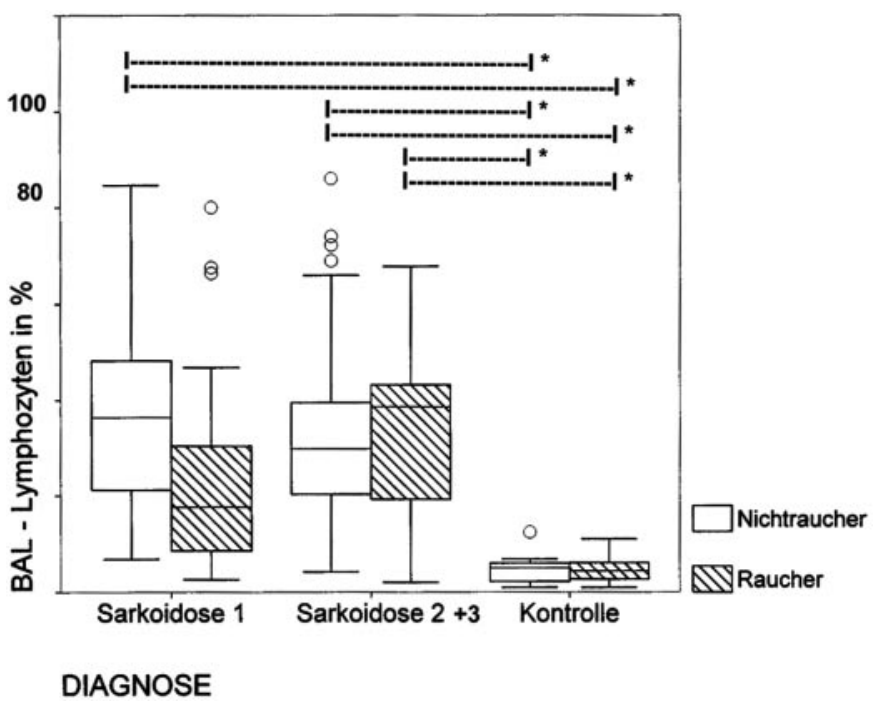

Abb. 2 Lymphozyten in \% der BAL-Zellen in Abhängigkeit von Stadium und Rauchverhalten. Boxplots mit Medianen und Interquartilenbereichen. $\bigcirc=$ Ausreißer. Signifikante Beziehungen $(p<0,05)$ mit l--------. ${ }^{*}$ markiert (Mehrfachvergleich nach Tukey). Die lymphozytäre Alveolitis ist bei R im Stadium I geringer ausgeprägt als bei NR.

Die prozentuale Verteilung der Lymphozytensubpopulationen wurde vom Rauchverhalten nicht beeinflusst. Auch die Wiederholung der Statistik mit den absoluten Zellzahlen änderte nichts an diesem Resultat. Der Albumingehalt der BAL war bei Rauchern signifikant geringer als bei Nichtrauchern.

\section{Diskussion}

In der vorliegenden Serie waren die Patienten mit Sarkoidose signifikant häufiger Nichtraucher als Raucher. Zwar war dieser Unterschied in der Kontrollgruppe nicht nachweisbar, aber die Häufigkeit und Geschlechtsverteilung der Raucher in der vorliegenden Serie wich nicht grundsätzlich von den Zahlen großer epidemiologischer Erhebungen ab. Beispielsweise wird in einem ATSStatement von 1996 eine mit unserem Kollektiv vergleichbare Raucherquote von $24 \%$ bei Frauen und $28 \%$ bei Männern in der Bevölkerung über 18 Jahre berichtet [10].

Dass es bei Rauchern im Vergleich mit den Nichtrauchern zu einer früheren Manifestation der Sarkoidose kommt, wurde bislang nicht beschrieben. Dieses Resultat der vorliegenden Serie kann nicht durch eine inhomogene Belegung der Krankheitsstadien, etwa durch Überwiegen der Nichtraucher in chronischen, fortgeschrittenen Stadien, erklärt werden. Denkbar wäre eine prinzipiell höhere ärztliche Untersuchungsfrequenz der Raucher aufgrund einer höheren nikotininduzierten Morbidität, so dass die Sarkoidose früher erkannt wird. Allerdings kann auch nicht ausgeschlossen werden, dass Inhalationsrauchen etwa durch Veränderung der pulmonalen Immunität eine frühere Manifestation der Sarkoidose begünstigt.

Die spirometrischen Leitgrößen waren nicht vom Rauchverhalten, sondern in erster Linie vom Stadium der Sarkoidose abhängig, wobei die Stadien II und III erwartungsgemäß stärkere Einschränkungen zeigten als das Stadium I. Stellt man aber eine Kor- 
Tab. 2 BAL-Lymphozytenpopulationen (Erläuterungen siehe Tab. 1)

\begin{tabular}{|c|c|c|c|c|c|c|c|c|c|c|}
\hline & \multicolumn{3}{|c|}{ Sarkoidose I } & \multicolumn{3}{|c|}{ Sarkoidose II und III } & \multicolumn{4}{|c|}{ Sarkoidose alle Stadien } \\
\hline & alle & $N R$ & $\boldsymbol{R}$ & alle & $N R$ & $R$ & alle & $N R$ & $R$ & $p$ \\
\hline CD3 (\%) & $\begin{array}{l}94,2 \\
\pm \\
4,1\end{array}$ & $\begin{array}{l}93,8 \\
\pm \\
4,4\end{array}$ & $\begin{array}{l}95,5 \\
\pm \\
2,8\end{array}$ & $\begin{array}{l}93,8 \\
\pm \\
5,2\end{array}$ & $\begin{array}{l}93,7 \\
\pm \\
5,3\end{array}$ & $\begin{array}{l}94,1 \\
\pm \\
5,0\end{array}$ & $\begin{array}{l}94,0 \\
\pm \\
4,7\end{array}$ & $\begin{array}{l}93,8 \\
\pm \\
4,9\end{array}$ & $\begin{array}{l}94,7 \\
\pm \\
4,2\end{array}$ & 0,25 \\
\hline CD4 (\%) & $\begin{array}{l}76,5 \\
\pm \\
12,6\end{array}$ & $\begin{array}{l}75,8 \\
\pm \\
12,5\end{array}$ & $\begin{array}{l}79,0 \\
\pm \\
13,4\end{array}$ & $\begin{array}{l}73,6 \\
\pm \\
16,7\end{array}$ & $\begin{array}{l}71,8 \\
\pm \\
17,2\end{array}$ & $\begin{array}{l}78,7 \\
\pm \\
8,7\end{array}$ & $\begin{array}{l}74,9 \\
\pm \\
14,4\end{array}$ & $\begin{array}{l}73,6 \\
\pm \\
15,2\end{array}$ & $\begin{array}{l}78,8 \\
\pm \\
10,7\end{array}$ & 0,16 \\
\hline CD8 (\%) & $\begin{array}{l}15,5 \\
\pm \\
9,9\end{array}$ & $\begin{array}{l}15,9 \\
\pm \\
10,0\end{array}$ & $\begin{array}{l}14,0 \\
\pm \\
9,9\end{array}$ & $\begin{array}{l}18,9 \\
\pm \\
13,7\end{array}$ & $\begin{array}{l}20,3 \\
\pm \\
14,6\end{array}$ & $\begin{array}{l}15,6 \\
\pm \\
10,1\end{array}$ & $\begin{array}{l}17,3 \\
\pm \\
12,2\end{array}$ & $\begin{array}{l}18,1 \\
\pm \\
12,8\end{array}$ & $\begin{array}{l}14,9 \\
\pm \\
9,9\end{array}$ & 0,34 \\
\hline$C D 4 / C D 8$ & $\begin{array}{l}8,9 \\
\pm \\
9,8\end{array}$ & $\begin{array}{l}8,3 \\
\pm \\
8,9\end{array}$ & $\begin{array}{l}10,9 \\
\pm \\
12,8\end{array}$ & $\begin{array}{l}7,0 \\
\pm \\
6,9\end{array}$ & $\begin{array}{l}6,2 \\
\pm \\
4,9\end{array}$ & $\begin{array}{l}9,3 \\
\pm \\
10,7\end{array}$ & $\begin{array}{l}7,9 \\
\pm \\
8,4\end{array}$ & $\begin{array}{l}7,2 \\
\pm \\
7,1\end{array}$ & $\begin{array}{l}10,0 \\
\pm \\
11,4\end{array}$ & 0,25 \\
\hline
\end{tabular}

relation zwischen dem Alter einerseits und der Lungenfunktion anhand des \%-Soll-Wertes der IVC andererseits her, zeigt sich bei den Rauchern eine signifikante negative Beziehung, die bei den Nichtrauchern nicht nachweisbar ist. Diese Zahlen ergeben somit keinen Hinweis darauf, dass Rauchen bei Sarkoidose einen protektiven Effekt auf den Lungenfunktionsverlauf ausübt und belegen gleichlautende Literaturmitteilungen [11,12].

Die BAL-Befunde, welche als diagnoserelevant bei Sarkoidose etabliert sind, zeigten zwischen Rauchern und Nichtrauchern keine signifikanten Unterschiede. Wohl könnte der Trend zu einer geringer ausgeprägten Lymphozytose bei Rauchern für einen bremsenden Effekt der Tabakrauchinhalation auf die bei Sarkoidose typische lymphozytäre Alveolitis sprechen. Ähnliche Beobachtungen wurden schon publiziert [7]. In die gleiche Richtung deutet das Resultat des Untergruppenvergleiches, welches bei Patienten mit Sarkoidose I nur bei Nichtrauchern, nicht aber bei Rauchern eine im Vergleich mit Kontrolle signifikante Lymphozytenvermehrung zeigt. Da im Gegensatz hierzu Patienten mit Stadium II + III unabhängig vom Rauchverhalten eine Lymphozytenvermehrung aufwiesen, wäre eine Stadienabhängigkeit des Raucheinflusses denkbar. Möglicherweise unterdrückt die Tabakrauchinhalation die lymphozytäre Alveolitis bei Sarkoidose im Stadium I stärker als in den Stadien II + III.

Die Lymphozytenpopulationen waren zwischen Rauchern und Nichtrauchern nicht verschieden. Dies steht im Gegensatz zu Mitteilungen supprimierender Effekte des Inhalationsrauchens auf die CD4-Zellen $[6,8,12]$. Weiterhin zeigte sich bei Rauchern ein geringerer Albumingehalt der BAL als bei Nichtrauchern. Auch hier ergaben sich Hinweise auf Unterschiede zwischen den Stadien dahingehend, dass Raucher im Stadium I eine stärkere Erniedrigung des Albuminspiegels aufweisen als in den Stadien II + III. Auch dies spricht dafür, dass die Tabakrauchinhalation in Abhängigkeit vom Stadium auf die Aktivität der Sarkoidose wirkt und im Stadium I die Entzündung stärker unterdrückt als in den Stadien II + III.

Die Aussagekraft der vorliegenden Studie wird durch die Zusammensetzung der Kontrollgruppe eingeschränkt. Neben deren geringem Umfang im Vergleich mit der Studiengruppe wiesen die
Kontrollen eine subjektive Hustensymptomatik auf. Allerdings konnte bei der im Vergleich mit den erkrankten Patienten identischen Diagnostik kein krankhafter Befund erhoben werden.

\section{Schlussfolgerung}

Die Mehrzahl unserer Patienten mit Sarkoidose waren Nichtraucher. Raucher waren im Mittel 5 Jahre jünger als Nichtraucher. Die Lungenfunktion wurde in erster Linie durch das Stadium der Sarkoidose und nicht vom Rauchverhalten bestimmt. Doch konnte ein negativer Effekt des Rauchens auf die spirometrischen Parameter mit zunehmendem Alter nachgewiesen werden. Raucher boten mit einem geringeren Albumingehalt und einem Trend zu einer geringer ausgeprägten Lymphozytose in der BAL Hinweise auf eine geringer ausgeprägte Alveolitis als Nichtraucher. Diese antientzündlichen Effekte erscheinen im Stadium I stärker ausgeprägt zu sein als in den Stadien II + III. Einflüsse des Rauchens auf die Verteilung der Lymphozytensubpopulationen waren nicht zu sehen.

\section{Literatur}

${ }^{1}$ Martinetti M, Tinelli C, Kolek V et al. "The sarcoidosis map": a joint survey of clinical and immunogenetic findings in two European countries. Am J Respir Crit Care Med 1995; 152 (2): 557-564

${ }^{2}$ American Thoracic Society/European Respiratory Society International. Multidisciplinary Consensus Classification of the Idiopathic Interstitial Pneumonias. Am J Respir Crit Care Med 2002; 165: 277- 304

${ }^{3}$ Donaghy M, Rees AJ. Cigarette smoking and lung hemorrhage in glomerulonephritis caused by autoantibodies to glomerular basement membrane. Lancet 1983; 17 (2): 1390-1393

${ }^{4}$ Murin S, Bilello KS, Matthay R. Other smoking-affected pulmonary diseases. Clin Chest Med 2000; 21 (1): 121 - 137

${ }^{5}$ Yamaguchi E, Okazaki N, Itoh A et al. Interleukin 1 production by alveolar macrophages is decreased in smokers. Am Rev Respir Dis 1989; 140 (2): 397-402

${ }^{6}$ Wallace JM, Oishi JS, Barbers RG et al. Lymphocytic subpopulations profiles in bronchoalveoläre Lavage fluid and peripheral blood from tobacco and marijuana smokers. Chest 1994; 105 (3): 847-852

${ }^{7}$ Drent M, Velzen-Blad H van, Diamant M et al. Relationship between presentation of sarcoidosis and Tlymphocyte profile. A study in bronchoalveolar lavage fluid. Chest 1993; 104 (3): 795-800 
${ }^{8}$ Laan M, Qvarfordt I, Riise GC et al. Increased level of interleukin-16 in the airways of tobacco smokers: relationship with peripheral blood lymphocytes. Thorax 1999; 54(10): 911 -916

${ }^{9}$ Costabel U. Atlas der bronchoalveolären Lavage. Stuttgart, New York: Thieme, 1994

${ }^{10}$ Cigarette smoking and health. Official statement of the American Thoracic Society. Am J Respir Crit Care Med 1996; 153 (5): 861 - 888
${ }^{11}$ Ström KE, Eklund AG. Smoking does not prevent the onset of respiratory failure in sarcoidosis. Sarcoidosis 1993; 10 (1): 26-28

12 Valeyre D, Soler P, Clerici C et al. Smoking and pulmonary sarcoidosis: effect of cigarette smoking on prevalence, clinical manifestations, Alveolitis, and evolution of the disease. Thorax 2000; 43 (8): 516-524
1. Die Deutsche Lungenstiftung verleiht jährlich im Rahmen der Jahrestagung der Deutschen Gesellschaft für Pneumologie einen Doktorandenpreis zur Förderung des wissenschaftlichen Nachwuchses.

2. Der Preis wird im Jahr 2003 erneut zweigeteilt ausgeschrieben. Es erfolgt eine Preisvergabe für die beste klinische sowie eine für die beste experimentelle Dissertationsarbeit auf dem Gesamtgebiet der Pneumologie.

3. Die Bewerber dürfen das 35. Lebensjahr nicht überschritten haben. Die wissenschaftlichen Grundlagen für die Arbeiten müssen in der Bundesrepublik Deutschland erarbeitet sein. Das Dissertationsverfahren sollte abgeschlossen sein, der Abschluss darf den Zeitraum von zwei der jeweiligen Jahrestagung der Deutschen Gesellschaft für Pneumologie vorausgehenden Jahren nicht überschreiten.

4. Der Preis wird von der Firma Boehringer Ingelheim Pharma $\mathrm{KG}$, Ingelheim/Rhein gestiftet und ist für beide zu prämierenden Arbeiten mit jeweils 2.000 Euro ausgestattet. Die beiden Einzelpreise können nicht geteilt werden.

5. Der Vorstand der Deutschen Lungenstiftung setzt ein Preiskomitee ein, das dem Vorstand der Deutschen Lungenstiftung aus den eingereichten Arbeiten die preiswürdigen benennt. Die Benennung erfolgt durch den Vorsitzenden des Komitees in Form eines schriftlichen Gutachtens, aus dem hervorgeht, warum der Arbeit der Preis zuerkannt wurde. Das Gutachten wird anlässlich der Preisverleihung durch den Vorsitzenden der Deutschen Lungenstiftung öffentlich vorgelesen.
6. Die Arbeiten müssen bis zum 15.01.2004 (Datum des Poststempels) beim Vorsitzenden der Deutschen Lungenstiftung, Herrn Prof. Dr. med. Helmut Fabel, unter folgender Anschrift eingereicht werden:

Deutsche Lungenstiftung e.V.

Prof. Dr. med. Helmut Fabel

Herrenhäuser Kirchweg 5, 3. OG

30167 Hannover

Die Arbeiten sind in deutscher Sprache abzufassen. Die Einreichung erfolgt in 4-facher Ausfertigung anonym unter Angabe des Alters, des Datums des Dissertationsabschlusses und unter einem Kennwort. In einem verschlossenen Umschlag mit Kennwort sind Name und genaue Anschrift anzugeben.

7. Die Bewerber können eine Woche vor Kongressbeginn das Kennwort der zum Preis ausgewählten Arbeit beim Vorsitzenden der Deutschen Lungenstiftung erfragen.

8. Der Doktorandenpreis der Deutschen Lungenstiftung wird im Rahmen der 45. Jahrestagung der DGP im März 2004 in Frankfurt am Main anlässlich der Abschlussveranstaltung verliehen. 